This item was submitted to Loughborough's Research Repository by the author.

Items in Figshare are protected by copyright, with all rights reserved, unless otherwise indicated.

\title{
Measurement and analysis of grip force during a golf shot
}

PLEASE CITE THE PUBLISHED VERSION

PUBLISHER

(C) Professional Engineering Publishing

LICENCE

CC BY-NC-ND 4.0

\section{REPOSITORY RECORD}

Komi, Erin R., Jonathan R. Roberts, and Steve Rothberg. 2019. "Measurement and Analysis of Grip Force During a Golf Shot". figshare. https://hdl.handle.net/2134/3577. 
This item was submitted to Loughborough's Institutional Repository by the author and is made available under the following Creative Commons Licence conditions.

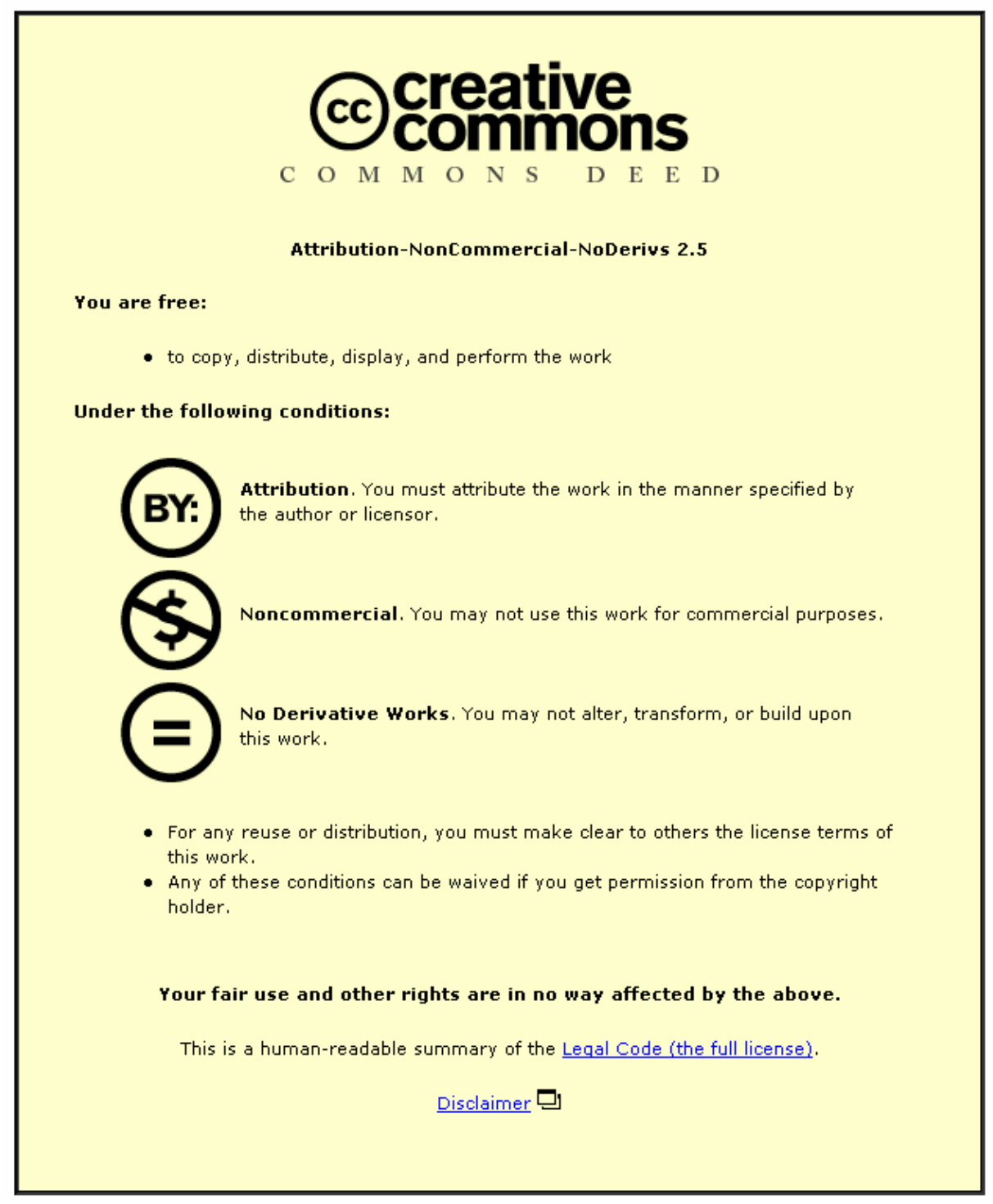

For the full text of this licence, please go to: http://creativecommons.org/licenses/by-nc-nd/2.5/ 


\title{
Measurement and analysis of grip force during a golf shot
}

E R Komi*, J R Roberts, and S J Rothberg

Wolfson School of Mechanical and Manufacturing Engineering, Loughborough University, Loughborough, UK

The manuscript was received on 11 December 2007 and was accepted after revision for publication on 25 March 2008.

DOI: 10.1243/17543371JSET9

\begin{abstract}
In this study, grip force was measured during a standard golf tee shot using two different measurement techniques. The first utilized a matrix-type thin-film sensor applied to a golf grip, from which total grip force could be readily determined. The second method involved 31 individual thin-film force sensors strategically placed on two golf gloves, allowing the force output of specific regions of the hands to be measured. Twenty golfers of varying ability participated in each test. The discovery of a unique grip force 'signature' for each golfer emerged from these data. That is, each golfer had a very repeatable total grip force trace, but these traces varied considerably between golfers. High-speed video was also recorded for many of the golfers tested so that key phases in the swing could be identified on the force traces.
\end{abstract}

Keywords: grip pressure, force sensors, pressure sensors, grip force signatures, golf swing

\section{INTRODUCTION}

There are many sports in which participants need to use an implement - bat, club, racket, etc. - to strike a ball or similar object. In such sports, the only point of contact that the participant has with the implement is at the grip. Only subtle differences in grip forces and wrist positions can affect shot distance and accuracy and may define the difference between a beginner and seasoned player or a high-level amateur and a professional [1].

In any grip action, a balance must be found between the force used to secure the object in the hand and wrist range of motion, as the two are inversely related (i.e. an increase in grip force produces a decrease in wrist range of motion) [2, 3]. Additionally, grip and pinch strength vary based on wrist angle [4-6]. In golf, this is especially important; a significant grip force is required to prevent the club from slipping out of the hands, while uninhibited wrist motion is desirable to create an accurate and repeatable shot and to maximize clubhead speed.

Grip force has previously been measured in a number of sports, including tennis, cricket, baseball, and also golf [7-14]. In golf, however, only limited

*Corresponding author: Wolfson School of Mechanical \& Manufacturing Engineering, Loughborough University, Loughborough, LE11 3TU, UK. email: E.Komi@lboro.ac.uk knowledge has been gained due to small sample sizes and force sensor selection. The most prominent work is that of Budney [7] and Budney and Bellow [8], who incorporated a hollow aluminium handle fitted with force sensors onto a steel-shafted driver. The force transducers used in this study were simply supported beam elements with metal foil, electrical resistance strain gauges and were strategically positioned to measure force in three locations. Grip force traces from amateurs and professionals were compared, but sample sizes were small.

Nikonovas et al. [15] developed a means to measure grip force similar to one of the methods described in this present paper. The golf shot was one of the example applications for the grip force system, but only one golfer was tested. The data from the golf test were comparable to the outcome of the work by Budney, and the grip force measurement system was shown to be suitable for the golf shot application. Further player testing with such a system would be required, however, to draw any general conclusions on grip force in a golf shot.

Opinions on the best way to grip a golf club vary greatly, with professionals and instructors disagreeing even on basics, such as which hand should control the club throughout the swing. Insight provided by scientists on how to maximize clubhead speed with optimally timed wrist torques [16] may have provided some indication, but the limited knowledge 
gained thus far on the grip forces produced during a typical golf shot have been insufficient to give clear answers to such basic questions.

This paper presents grip force profiles for a large selection of golfers of varying ability, and trends related to how various golfers grip the club are discussed. Two methods of measuring grip force are utilized such that both total force and the forces at individual regions of the hands could be monitored. This information represents the most extensive experimental study on grip force in golf to date.

\section{METHODS}

\subsection{Sensor selection}

Thin, flexible force sensors were selected for measurement of grip force in golf for several reasons. They are extremely thin and lightweight, they can bend to fit around the curve of the golf grip or be attached to gloves, and they allow measurement of time-varying force at numerous locations simultaneously. Three types of force sensor were rigorously tested to determine their suitability for this application; player tests involving two of the sensor types are described in this paper. The two chosen sensors were found to have static accuracy, hysteresis, and drift errors (at $1 \mathrm{~s}$ after load application) of 7-10 per cent, 6 per cent, and 2-4 per cent, respectively, and dynamic accuracy errors of around 20 per cent for Flexiforce sensors and 60 per cent for Tekscan 9811 sensors [17].

The sensors used for this study were both manufactured by Tekscan, Inc. (Boston, MA). These force sensors use a semi-conductive ink that is applied between electrical contacts and thin polyester sheets, giving the sensors a resultant thickness of $0.1 \mathrm{~mm}$. They respond to a change in applied force with a linear change in resistance for which the sensor can be calibrated (linear $R^{2}=0.94$ ). The sensors are available in a number of configurations; for this study, a single load cell (Flexiforce) with a force rating from $0-111 \mathrm{~N}(25 \mathrm{lbf})$ and a matrix arrangement (9811) with a pressure rating of $0-517 \mathrm{kPa}$ (75 psi) were used, as shown in Fig. 1. The 9811 sensor has a $6 \times 16$ matrix of sensing cells, resulting in a total of 96 sensing elements.

\subsection{Sensor configurations for tests}

The size and shape of the two sensor types were primary factors in determining how they should be placed at the hand-grip interface for measurement purposes. The Tekscan 9811 matrix sensor was best suited to measuring total grip force and was attached directly to the golf grip. To facilitate this task, the sensor was cut between each column, leaving six strips of sensing elements, connected at one end, which were evenly spaced running down the length of the golf club grip. Double-sided tape was used to attach the sensor to the rubber golf grip and thin strips of electrical tape were positioned over the non-sensitive areas of the sensor to hold it in place. Micropore tape (a permeable, non-woven, surgical, synthetic adhesive manufactured by $3 \mathrm{M}$ ) was wrapped around the sensor to help protect it from damage caused by shear forces produced during a golf shot. The use of Micropore tape and limiting the number of shots taken with a particular sensor prevented problems with sensor durability that had been noted in previous tests [17]. When attached to the grip, 84 of the 96 sensing elements rested entirely against the grip surface and outputs from those elements were considered in all analyses. Images of the sensor on the grip are displayed in Fig. 2. The force output from Tekscan 9811 sensors were monitored with a complete, commercially available acquisition system provided by the manufacturer for this sensor type. The 9811 sensors have passive regions between the active sensing elements, but this is compensated

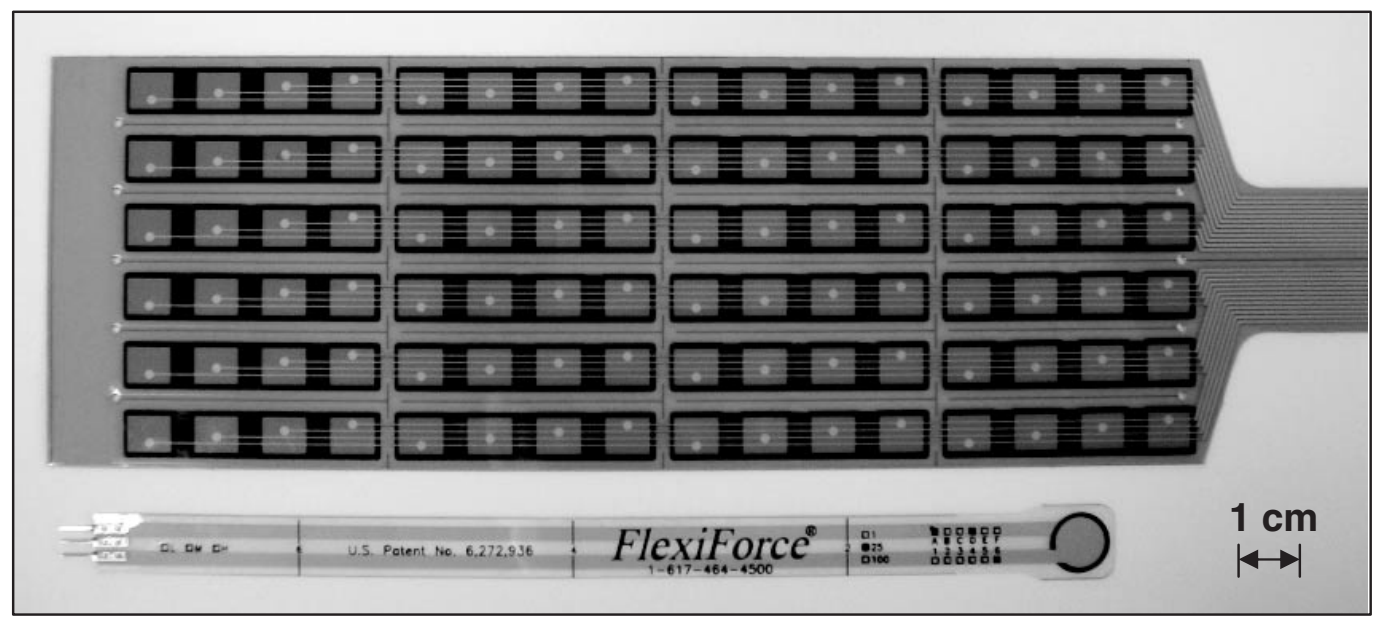

Fig. 1 Tekscan 9811 matrix and Flexiforce single-cell sensors 


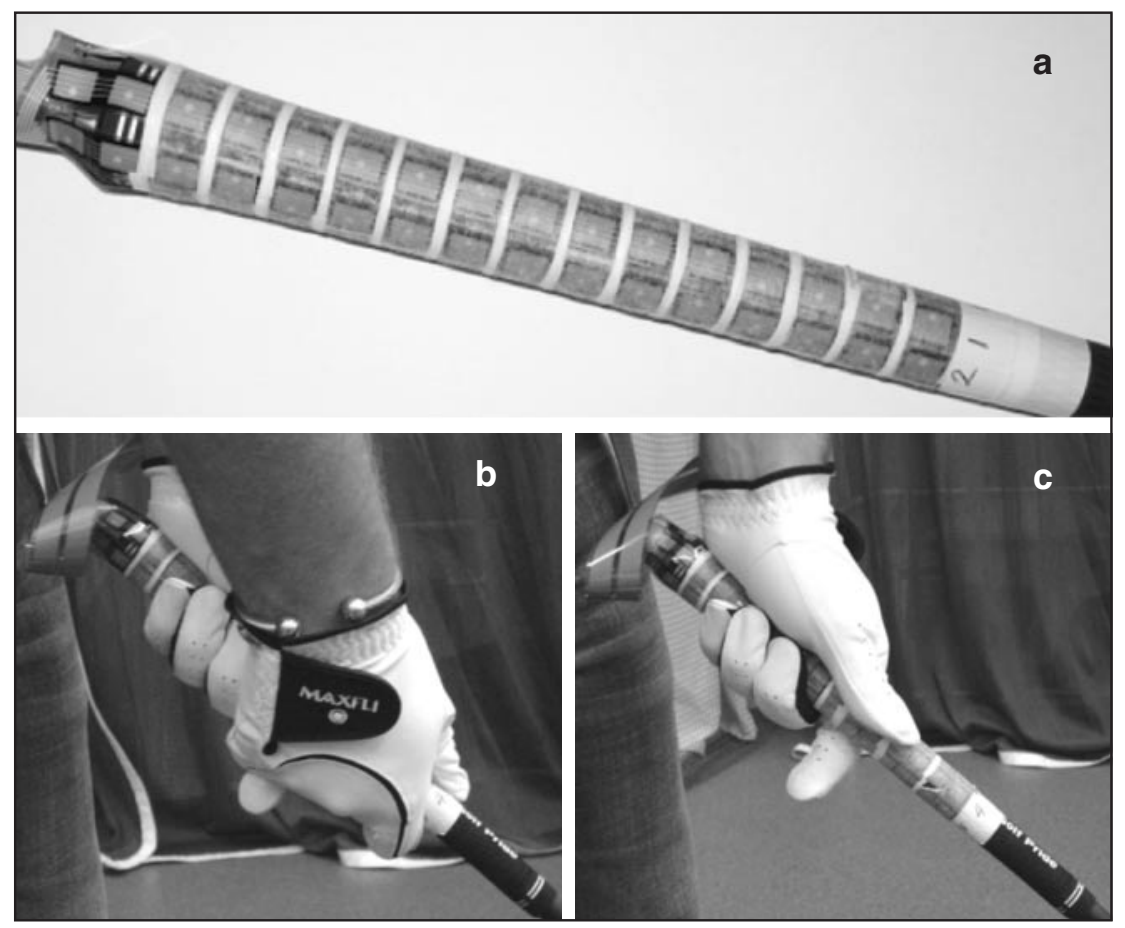

Fig. 2 Tekscan 9811 sensor on grip

for in the Tekscan software and no further adjustment of the force outputs is required.

Being a single load cell type sensor, Flexiforce has the benefit that any number of sensors can be used simultaneously and be placed independently of one another. For this reason, the sensors were attached to golf gloves so that force could be monitored at key regions of both hands. For the golf grip force task, a 32-channel data acquisition system with 16-bit A/D resolution was employed with one channel reserved to record sound data (in order to determine the moment of impact) and all others were connected to force sensors. The 31 force sensors were then attached to strategic locations on two golf gloves, as shown in Fig. 3. These regions were chosen based on areas of peak loading measured during preliminary tests and on the recommendations of previous researchers $[\mathbf{7}, \mathbf{8}, \mathbf{1 5}]$. Similar sensoron-glove configurations have been used in the past to measure grip force $[\mathbf{1 5}, \mathbf{1 8}]$. Double-sided and Micropore tape were again used to help position, and secure and protect the sensitive area of each sensor. The remainder of each sensor was directed along the most convenient route to the back of the hand using hand-sewn loops of elastic thread as a guide.

\subsection{Test protocol}

Twenty right-handed golfers of varying ability (male and female, handicaps ranging from $0-22$, with two players new to the game and without handicap) participated in each test. Before testing, they were

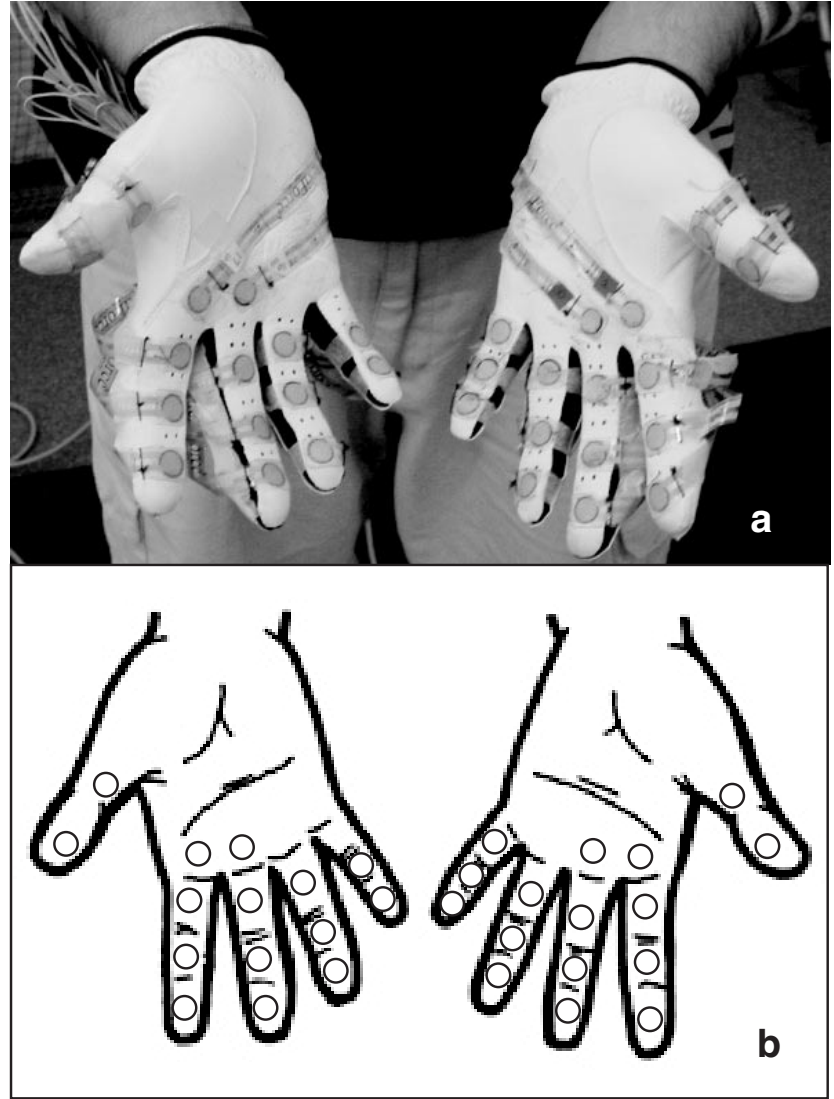

Fig. 3 (a) 31 Flexiforce sensors attached to two gloves, and (b) sensor locations on the hands 
allowed to warm up for as long as they liked. For the test, the golfers all wore two gloves (cabretta palm and synthetic leather top). All cables or wires connected to the force sensors were directed along the golfers' arms and over their shoulders. Time was given for the golfers to adjust to the feel of the sensors and cables. During the test, each golfer hit 10-12 shots with a standard driver (graphite shaft, titanium head, $9.5^{\circ}$ loft) in an indoor netted enclosure off an artificial turf matt with rubber tee. Sensor durability concerns emerged in previous work [17] and sensors were consequently changed frequently (every three to four golfers) to avoid this problem. These tests complied fully with the Loughborough University Ethical Advisory Committee code of practice for investigations with human participants.

Besides grip force, impact sound was also measured during each test in order that impact time could be determined on the force traces. For the Tekscan 9811 test, light gates were used to trigger both the force sensors and a sound level meter at the start of the backswing, which were sampled at 264 and $500 \mathrm{~Hz}$, respectively. The sampling rate was increased for the Flexiforce test to increase the likelihood of capturing the moment of impact in the force trace, although resultant file sizes were also taken into consideration. In the Flexiforce test, the sound level meter and force sensors were triggered manually through the same data acquisition system, and all channels were sampled at $1 \mathrm{kHz}$.

To further relate the grip forces measured to key phases during each golf shot, 18 of the 20 golfers who participated in the test with Flexiforce sensors on golf gloves also had their swing captured on high-speed video. Two shots were recorded for each golfer using a Photron Fastcam Ultimate APX highspeed video camera sampling at 500 frames per second. For each player, the start of takeaway, start of downswing, impact time, and end of followthrough were determined, and the total downswing and follow-through times were calculated as the mean from the two recorded shots. Identification of all of these points in the swing, excluding impact time, is somewhat subjective, and it is estimated that each point is within \pm 3 frames ( $\pm 6 \mathrm{~ms}$ ). It was noted that downswing time for each golfer was the most consistent part of the swing, having the lowest standard deviation (SD).

\section{RESULTS}

Each player's grip force was recorded for 10-12 shots with each sensor arrangement. The total grip force was then computed for each shot by summing the outputs from all sensing elements (either on the grip or on gloves). The exact moment of impact was determined for each trace from the sound measurement and denoted as time $=0 \mathrm{~s}$, and every shot for a particular golfer was aligned at this point.

\subsection{Grip force 'signatures'}

The data collected from the 9811 sensors on the golf grip were used primarily to evaluate total grip force produced by the golfers tested. The outputs from 84 sensing elements situated on the grip were used for the total force computation. After aligning the total force traces based on moment of impact, as illustrated in Fig. 4, it was observed that each golfer had their own unique grip force 'signature'. The force traces for an individual golfer were very repeatable, but differed from those of other golfers. All 20 golfers were found to have a grip force signature, including the two subjects that were new to the game and had only started playing six months prior to the test, labelled as NH (no handicap).

In order to examine the grip force signatures more closely, a cross-correlation technique was used to compare the total force profiles of the 20 golfers. The aim of this cross-correlation was to give an indication of the repeatability of an individual golfer's force trace and to quantify the similarity between different golfers. A 2-s segment of the recorded force traces, from $0.75 \mathrm{~s}$ prior to impact through to $1.25 \mathrm{~s}$ after impact (for a total of $J=528$ data points for the 9811 tests), was used in all calculations. Each force trace for subject $m$ and shot $p$ was normalized as shown in equation (1), where the mean of that trace was subtracted from each point of that trace, and this difference was then divided by the SD. A mean normalized trace for each golfer was computed using equation (2), where $P$ is the total number of shots taken by that golfer. Finally, the crosscorrelation was computed between the normalized force for the $p$ th shot of player $m$ and the normalized mean for player $n$, and this was, itself, normalized by the autocorrelation of the force profile for player $n$, as seen in equation (3). This cross-correlation value was actually an estimate of the peak crosscorrelation calculated for the zero delay between the individual trace of player $m$ and the mean trace for player $n$ based on the traces having been aligned by the moment of impact. The normalized traces were used in this case in order to highlight variations in profile shape rather than absolute force values. Values of the cross-correlation typically lie between 0 and 1 , where higher numbers indicate a stronger correlation.

$$
\tilde{f}_{m p}(j)=\frac{f_{m p}(j)-\bar{f}_{m p}}{\sqrt{\operatorname{var}\left(f_{m p}\right)}}
$$




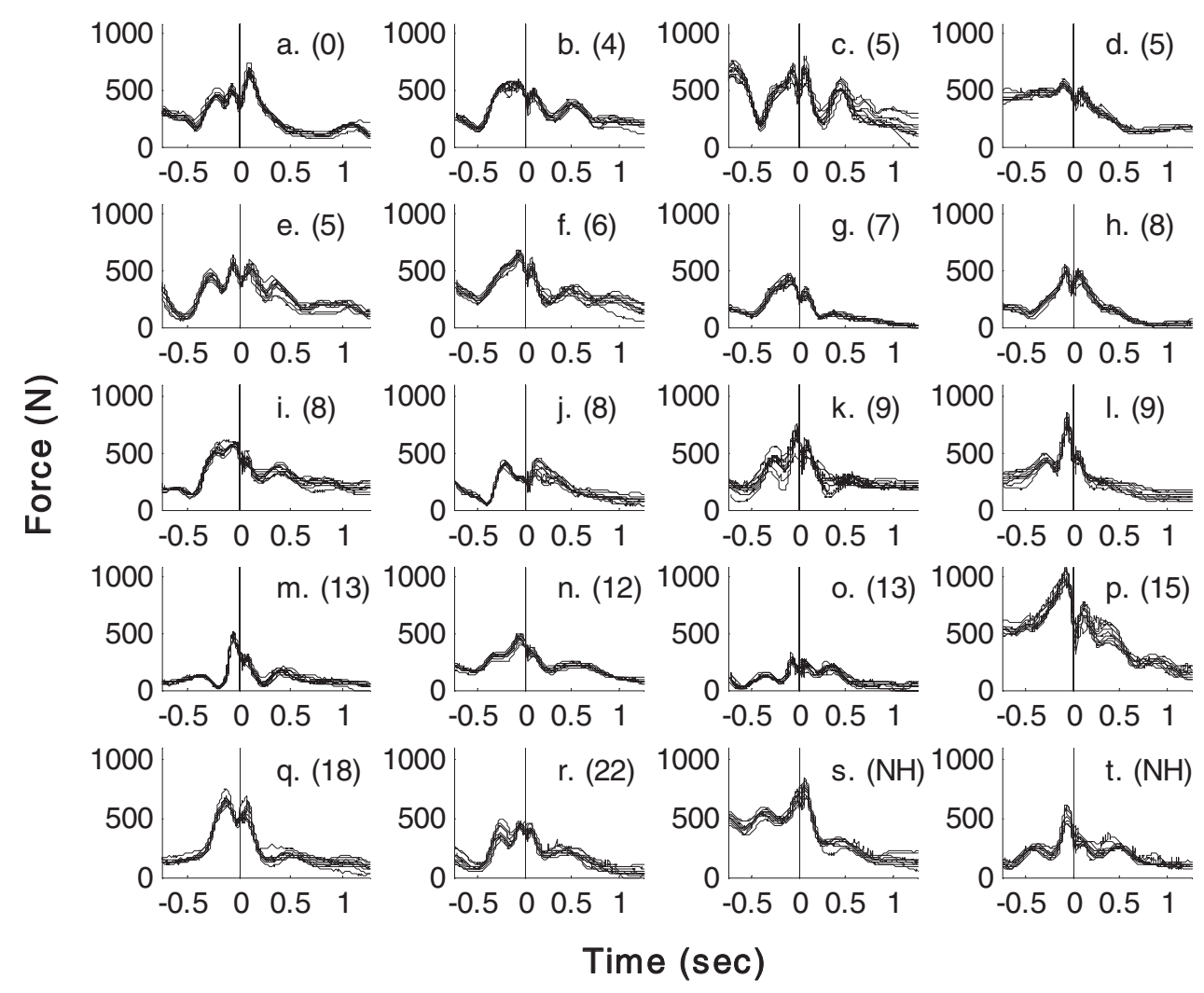

Fig. 4 Total force from 10 shots for each of 20 golfers ('a.'-'t.') with a 9811 sensor on grip, all shots aligned at impact (time $=0 \mathrm{~s}$ ), handicap shown in parentheses

$$
\begin{aligned}
\tilde{F}_{m}(j)= & \frac{1}{P} \sum_{p=1}^{p=P} \tilde{f}_{m p}(j) \\
\hat{\gamma}_{n, m p}= & \frac{\sum_{j=1}^{j=J} \tilde{F}_{n}(j) \tilde{f}_{m p}(j)}{\sum_{j=1}^{j=J} \tilde{F}_{n}(j) \tilde{F}_{n}(j)}
\end{aligned}
$$

All 200 total force traces from individual shots were compared to the 20 mean force curves, one for each golfer, using this method. With $P=10$ shots, this resulted in 10 normalized cross-correlations for each combination of players, from which an average was taken. In each case, as expected, the crosscorrelation is highest when the force traces of a particular golfer are compared to the mean trace for that same golfer (all greater than 0.95). There was only one occasion where such high correlation was reached when the signatures from two different golfers were correlated.

Although it is difficult to split the various grip force signatures into categories with only 20 golfers tested, a few trends do appear to emerge. In all cases, impact occurs near a local minimum, with local maxima on either side. A number of golfers have a fairly defined double peak occurring with impact lying somewhere in the middle, and, in many cases, the comparisons between golfers with these double-peak profiles produced the higher cross-correlation values. According to the correlation, the two players that have the most similar total grip force profiles are shown in plots 'f.' and 'g.' of Fig. 4 ( $\hat{\gamma}=0.95)$. These two players are included in a group of four ('f.', 'g.', 'n.', and 'q.') who all have high cross-correlations with one another (above 0.9). Another group with high crosscorrelations is 'p.', 'g.', and 'l.', suggesting the existence of families of grip force signatures. It is interesting to note that some of the higher handicap golfers have less defined peaks surrounding impact.

Some additional comments can be made about the magnitudes of the total force measurements. The peak values ranged from around 300-1100 N, with all category 1 golfers in the range of 600-750 N. The four female golfers ('b.', 'n.', 'o.', and 't.' in Fig. 4) tended to have peak values in the mid to lower region from about 300-600 N.

The existence of grip force 'signatures' was again verified using data from the Flexiforce tests. Representative individual total force curves from three golfers were aligned by impact and plotted to demonstrate this in the left column of Fig. 5. In the right column of the same figure, the summed force from the two sensors on the left thumb of each golfer are displayed to show the repeatability of the force 

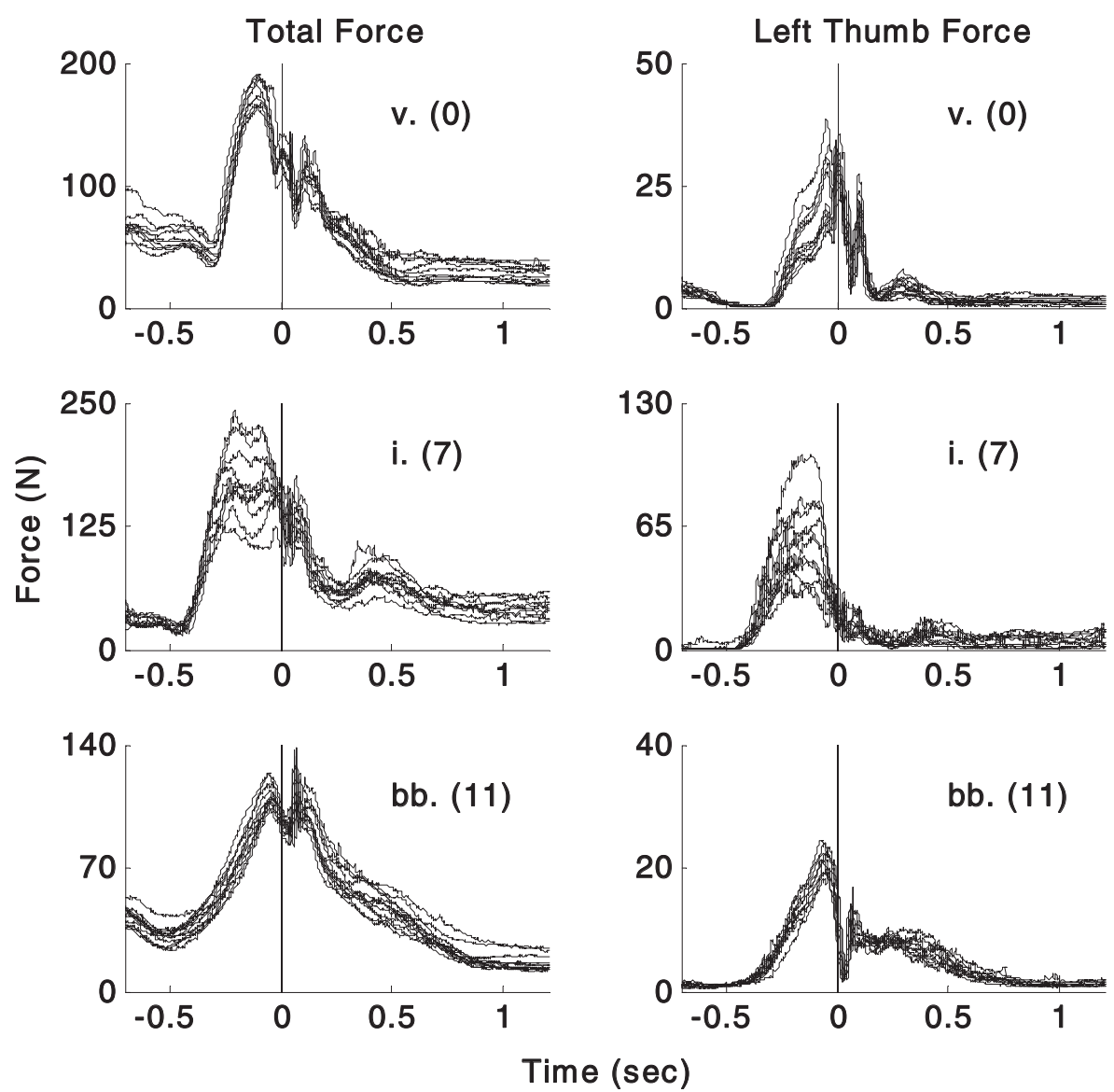

Fig. 5 Twelve total (left column) and left thumb (right column) force traces for three golfers as measured by Flexiforce sensors; all shots aligned at impact (time $=0 \mathrm{~s}$ ), handicap shown in parentheses

produced at individual regions of the hands as well. The left thumb traces showed a little more variability, particularly for golfer 'i.', but this may be partially due to small shifts in the positioning of sensors relative to the hands between shots. A cross-correlation was conducted using the methods described previously to compare the mean total force for these three golfers with the individual total force curves for each particular golfer. It was found that the individual total force curves for each golfer were highly correlated with the mean for that golfer, with mean cross-correlation values of $0.985,0.976$, and 0.993 for golfers 'v.', 'i.' and 'bb.' respectively.

Mean total force was also computed for each of the 20 golfers that took golf shots with Flexiforce sensors applied to golf gloves. The mean traces were used so that on occasions when a single sensor did not work properly during a test (typically evidenced by a sharp spike in the data), the output from that sensor could be removed rather than having to eliminate an entire measurement. The force traces for the golfers that participated in both sets of tests were very similar, but the amplitude of the output from the Tekscan 9811 test was consid- erably higher than that from the Flexiforce sensors, as the 9811 matrix covered effectively all of the hand-grip interface while the Flexiforce sensors intentionally covered a smaller proportion. The mean total force traces from the two tests were therefore normalized by subtracting the mean and dividing by the SD for the purpose of comparison, as shown in Fig. 6. The normalized force traces from the two tests are very similar $(\hat{\gamma}>0.87)$, which indicates a high level of consistency by the golfers as these two tests were conducted several months apart.

\subsection{Individual finger forces}

The Flexiforce sensors were used on golf gloves with the intention of measuring grip force at known locations on the hands. This allowed the force contribution for specific regions of the hands to be analysed individually. The total, left hand and right hand forces were compared between the 20 golfers to identify similarities using the cross-correlation method. This time, however, only mean force traces were used in the cross-correlation. The total, left 


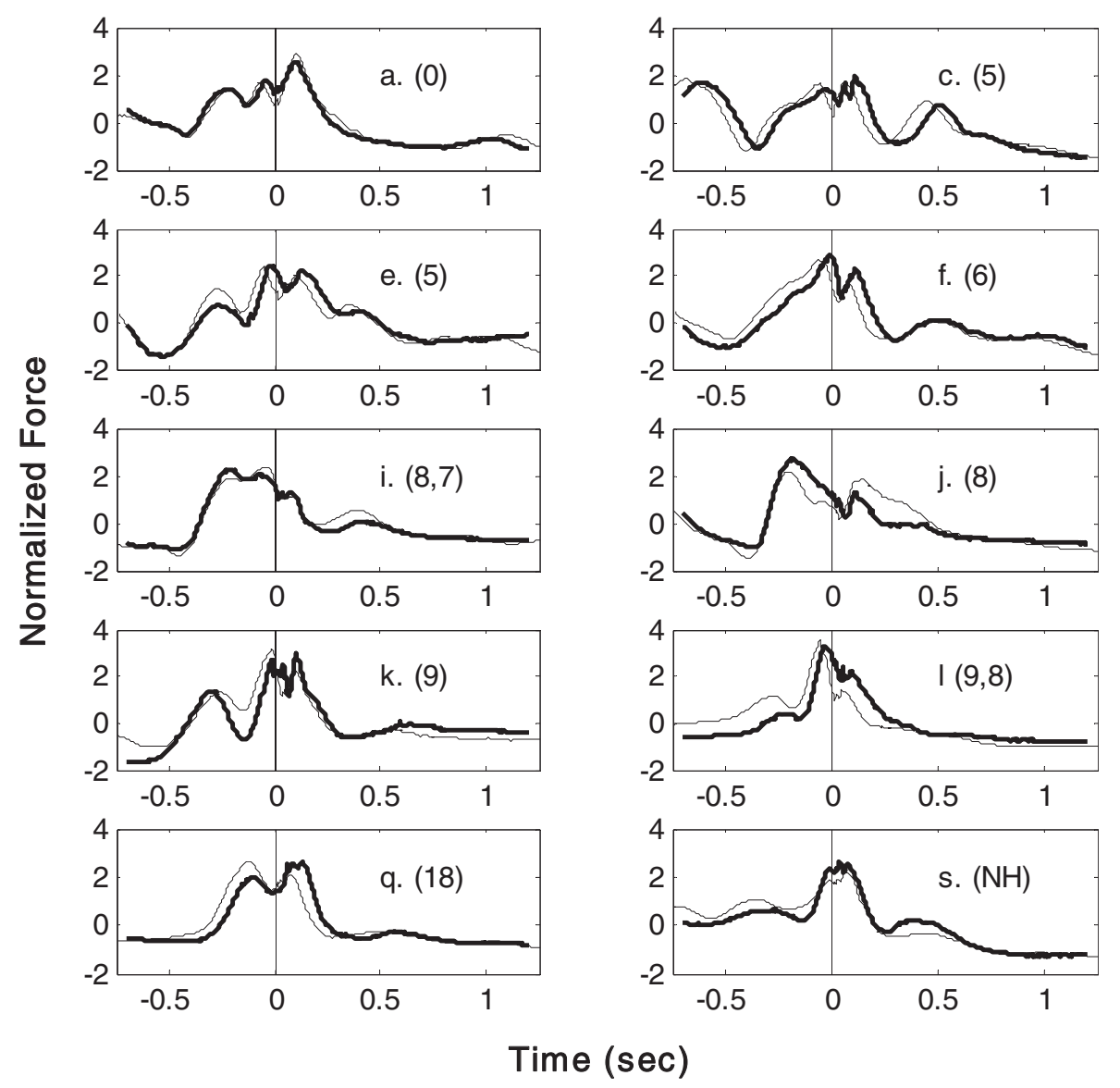

Fig. 6 Normalized mean total force traces measured with -9811 and - Flexiforce sensors; all shots aligned at impact (time $=0 \mathrm{~s}$ ), handicap shown in parentheses (note that the handicap of golfers 'i.' and 'l.' decreased between tests)

hand and right hand traces for eight of the 20 golfers tested are shown in Figs 7(a) and (b), with the handicap for each golfer in parentheses above the plot along with a one- or two-letter identification for each test subject. This identification carries through from the previous test (9811 sensor on the grip) so that golfers who participated in both tests can be identified.

From the Flexiforce test total force cross-correlation, it was found that the pair of golfers with the highest correlation with one another were 'a.' and 'y.'. In this case, it was not just the location of the primary peaks that caused the high correlation between these golfers; each had a fairly steady increase in force starting about $.4 \mathrm{~s}$ before impact and a peak after impact.

The cross-correlation of the left hand forces indicated that there are several groups of golfers that use their left hand in a similar fashion. One such group involved golfers 'a.', 'e.', and 'y.' which includes two of the golfers with the highest total force cross-correlation value. The total left hand forces for these three golfers can be seen in Fig. 7(a). The three golfers all apply an early force with their left hand that is approximately maintained until impact, with an additional peak just after impact, followed by a decrease in force until around the end of their follow-through. It is interesting to note that golfers 'a.' and ' $y$.' have high cross-correlation values for both total and left hand forces, while golfer 'e.' has a high correlation with 'a.' and 'y.' for left hand force alone.

The cross-correlation values for the right hand revealed that there was a much greater variation in the way that the golfers tested gripped with this hand. Very few players had a $\hat{\gamma}>0.9$, with another golfer. One group of golfers did exhibit a higher level of correlation, and they include 'q.' and 'bb.' (see Fig. 7(b)). During the downswing, each of these golfers maintained a steady right hand force, which began to increase prior to impact, and peaked after impact before dropping down again. An additional, smaller peak occurred at approximately the end of followthrough.

Figures 7(a) and (b) (plots B and C) displays individual finger and palm forces of the left and right hands for some of the golfers tested. There is great variation between golfers, but, again, a few trends can be 
a. (0)
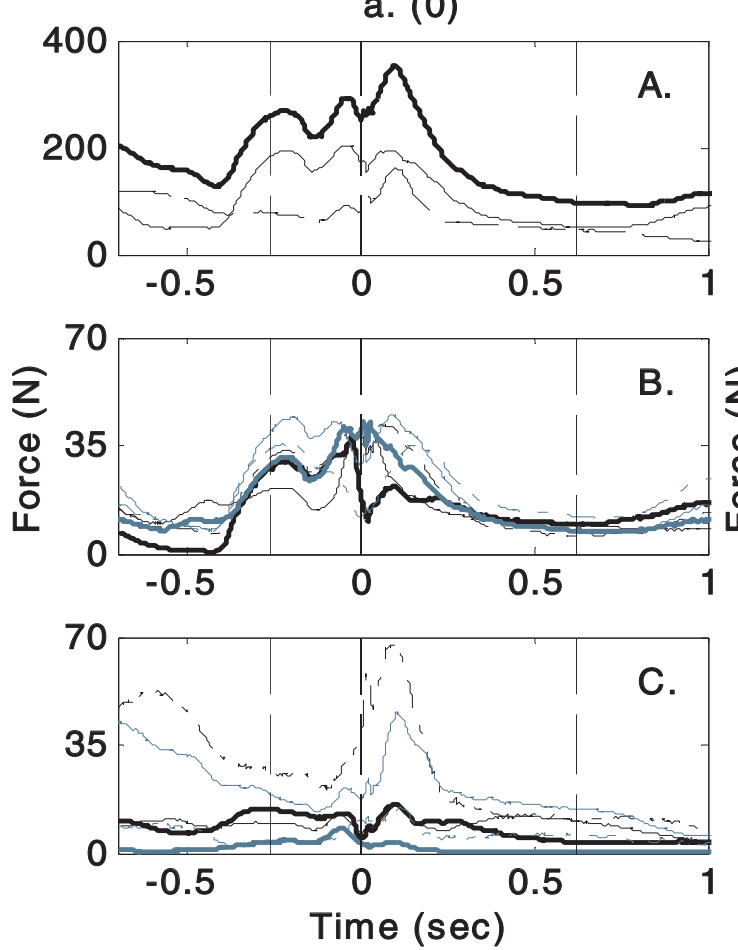

e. (5)
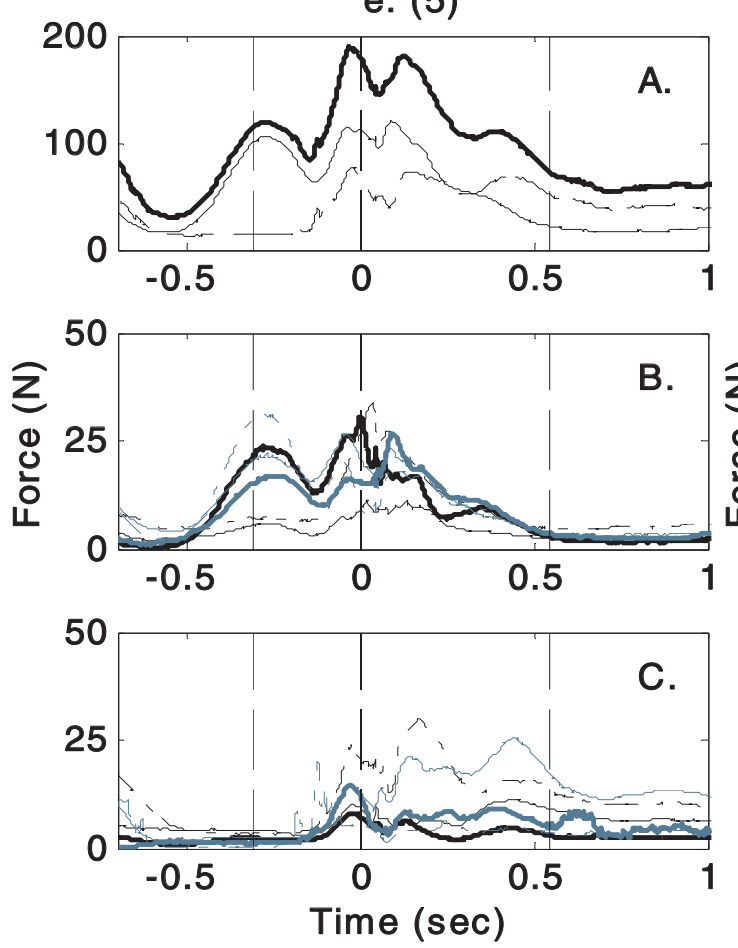

v. (0)
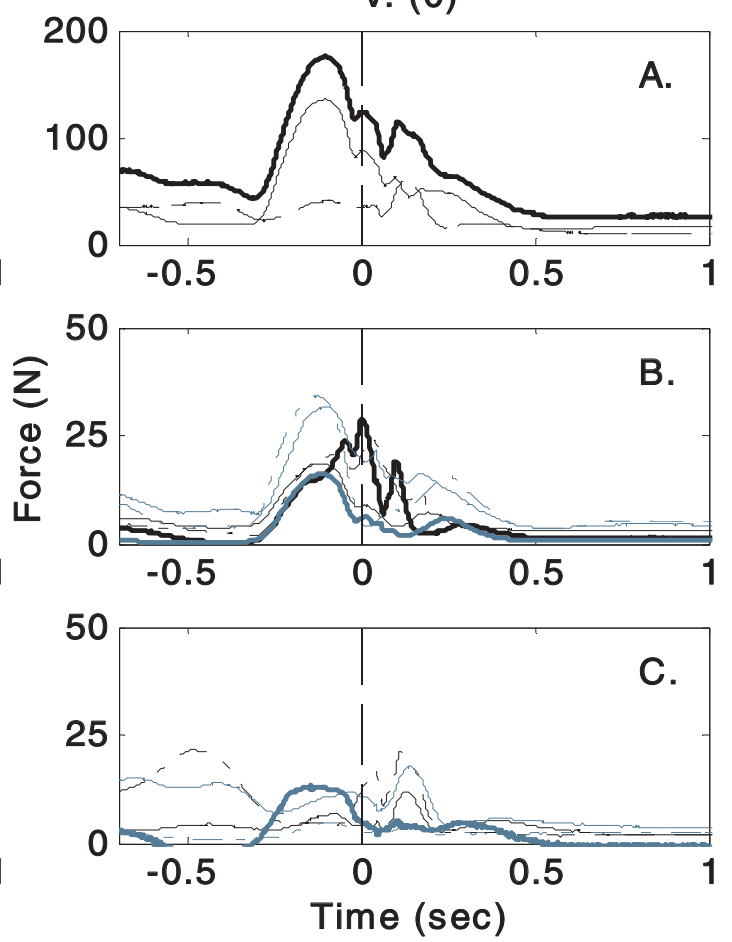

y. (6)
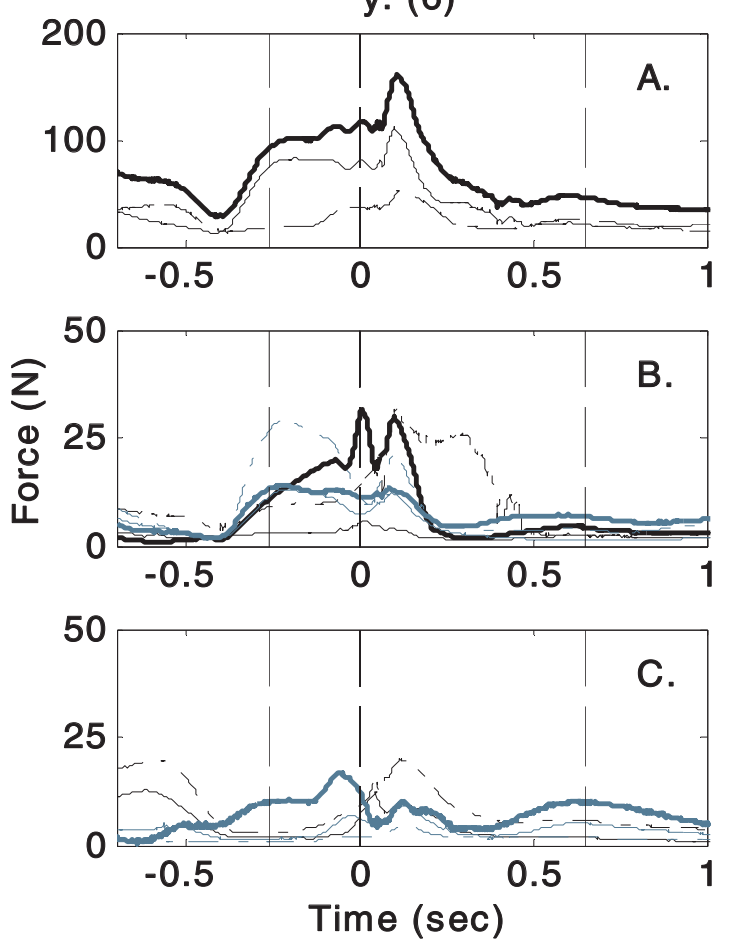

Fig. 7(a) Golfer grip forces measured by Flexiforce sensors with all shots aligned at impact (time $=0 \mathrm{~s}$ ) and handicap is shown in parentheses; (A.) mean total (-), left hand (-) and righthand (--) forces , (B.) and (C.) forces from regions of the left and right hands, respectively: thumb (-), index finger $(-)$, middle finger $(---)$, ring finger $(-)$, little finger $(--)$, and palm $(-)$. The approximate start of downswing and end of follow-through are also indicated by vertical lines before and after impact, respectively 
i. (7)
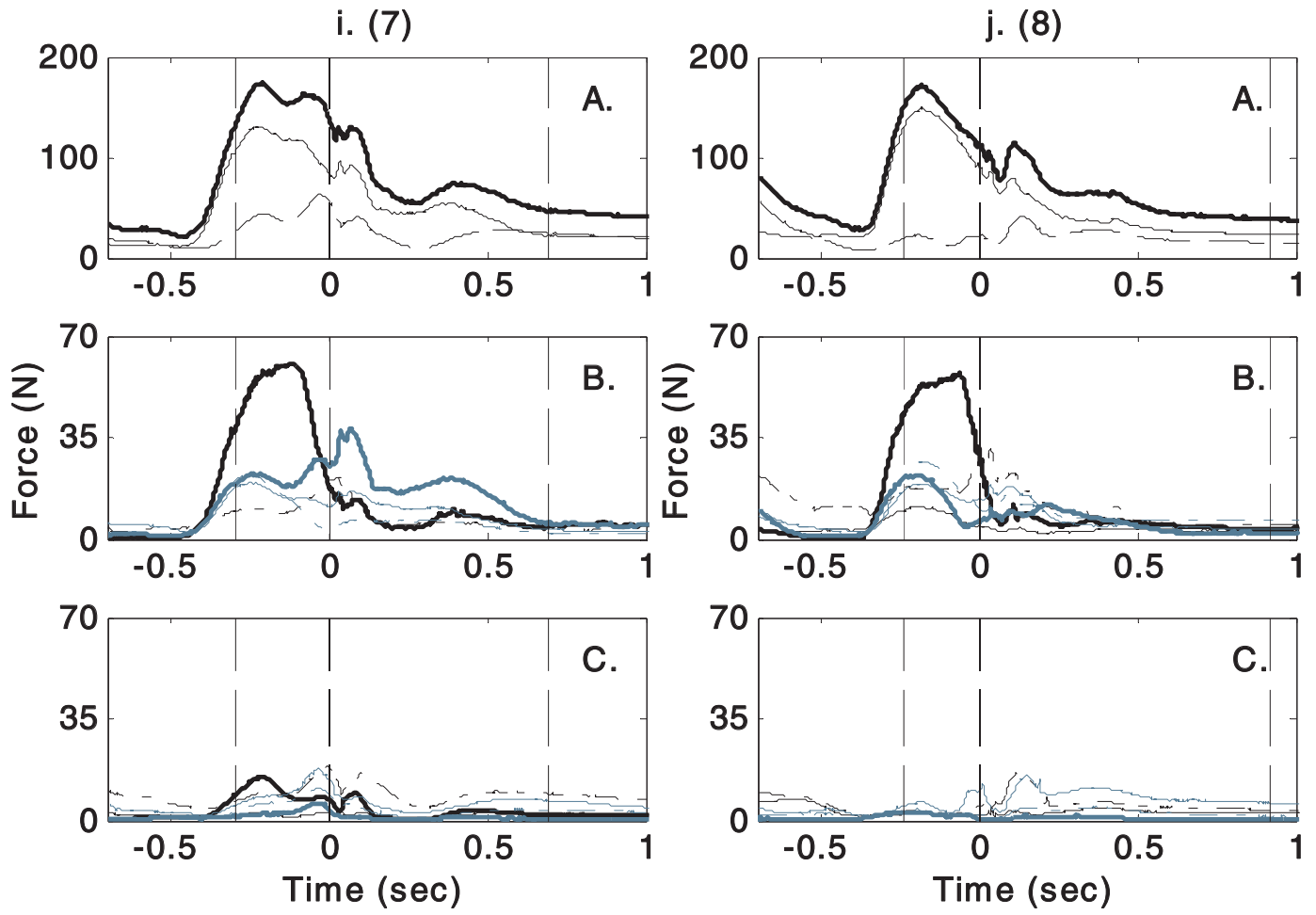

bb. (11)
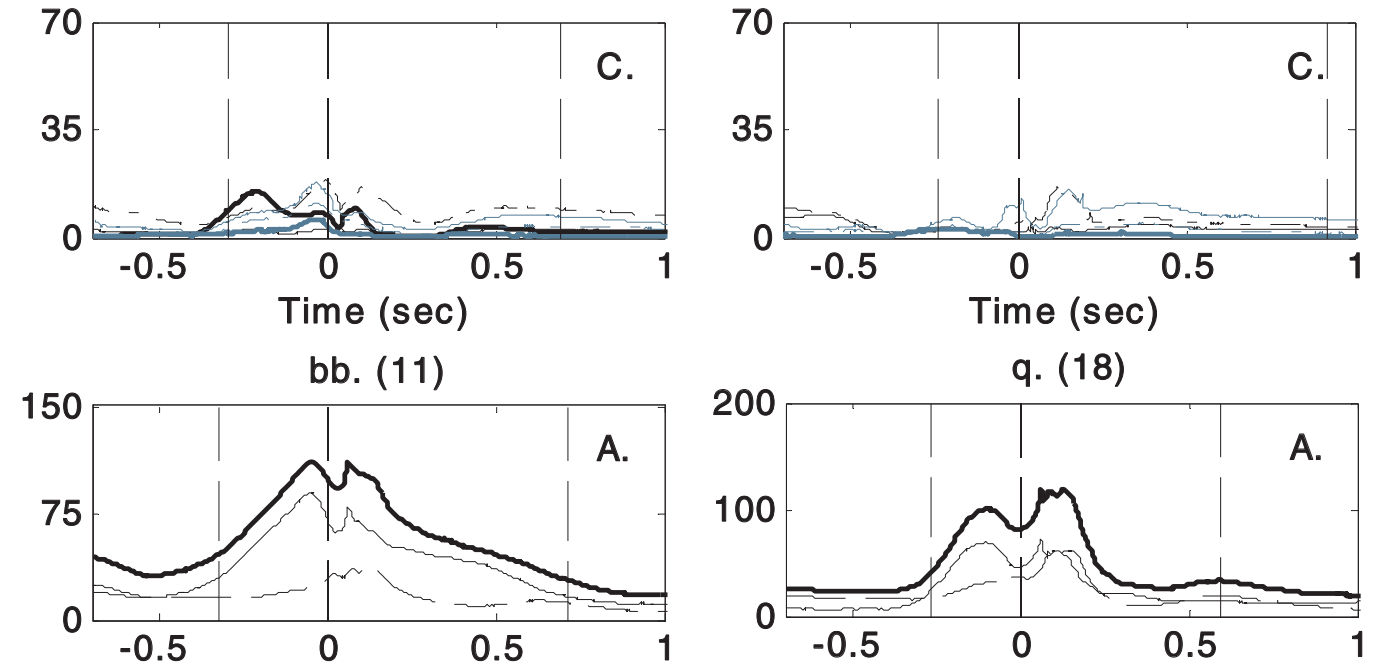

q. (18)
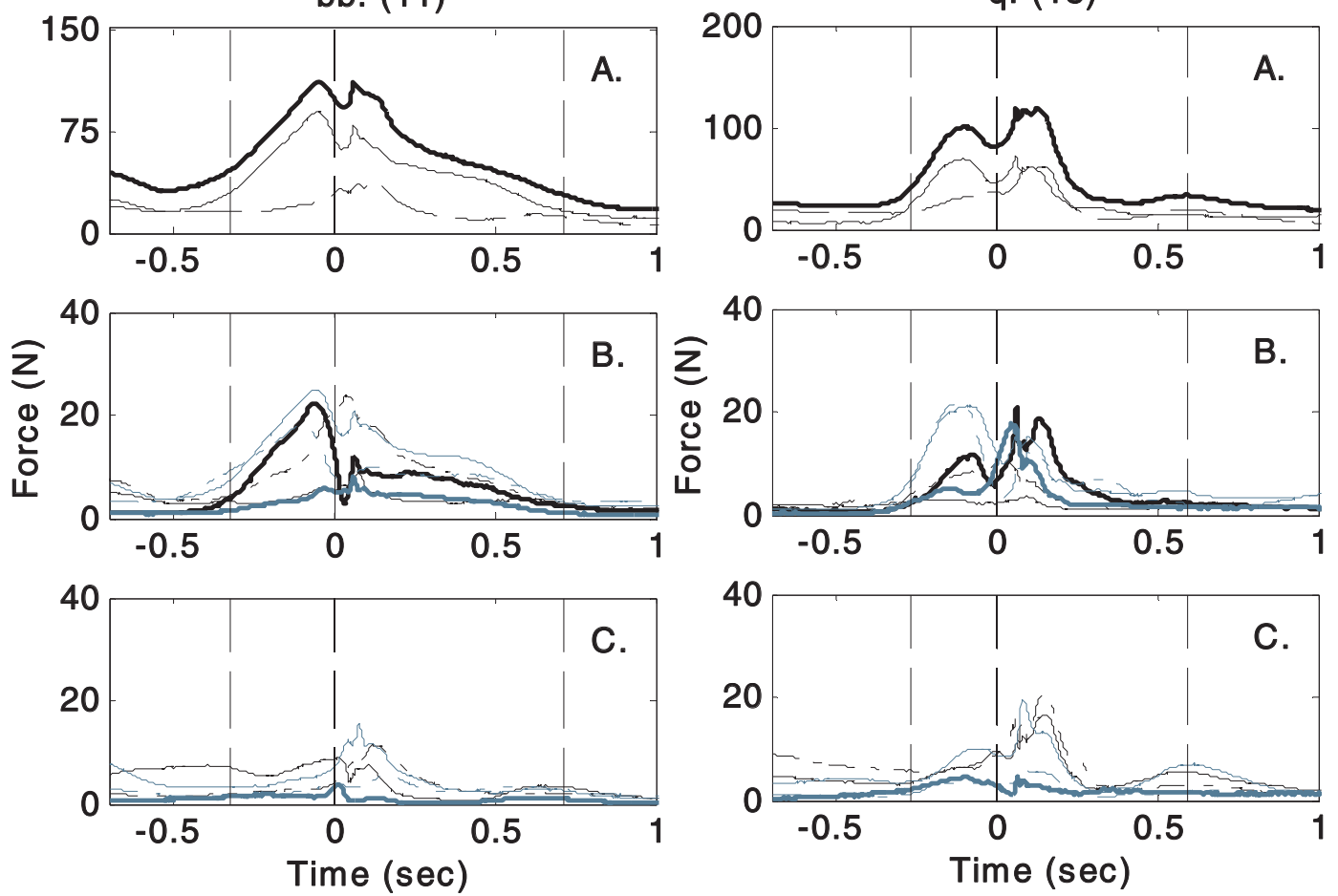

Fig. 7(b) Golfer grip forces measured by Flexiforce sensors with all shots aligned at impact (time $=0 \mathrm{~s}$ ) and handicap is shown in parentheses; (A.) mean total (-), left hand (-) and right hand (---) forces, (B.) and (C.) forces from regions of the left and right hands, respectively: thumb (-), index finger $(-)$, middle finger $(---)$, ring finger $(-)$, little finger $(--)$, and palm $(-)$. The approximate start of downswing and end of follow-through are also indicated by vertical lines before and after impact, respectively 
observed. For the left hand, there was often a dominant peak before impact produced either primarily by the thumb (golfers 'i.' and ' $j$.') or a combination of the ring and little fingers (golfers 'q.', ' $v$.' and 'y.'). Additionally, a large number of golfers appeared to have a more even distribution of force over their left hand, with peaks before and after impact containing contribution from all parts of the hand (golfers 'a.', 'e.', and 'bb.'). For the right hand, it appeared that many golfers used their middle and ring fingers to control the club during part of the take-away and backswing evidenced by peaks early in the trace (golfers 'a.', 'i.', 'j.', 'v.', and 'y.'). Additionally, a large number of golfers had peaks just after impact using one or more of the index, middle and ring fingers (golfers 'a.', 'i.', 'j.', 'q.', 'v.', and 'y.'). Otherwise, the force outputs from the various regions of the right hand tend to be similar.

\subsection{High speed video}

Based on the results of the high-speed video recordings, the approximate start of downswing and end of follow-through were also plotted with the force traces for seven of the golfers shown in Figs 7(a) and (b). With this additional information, it is also possible to see where in the swing various peaks occur, and this helps to compare traces from the different golfers to give a better idea of the timing. It was interesting to note that the first peak for the double-peak golfers occurs at different moments in the swing. For golfers 'i.' and 'j.', the peak occurred at or just after the start of the downswing, golfer 'q.' had the first peak mid-way through the downswing, and with golfers 'e.' and 'bb.' the first peak occurred at or near impact. For these doublepeak profiles, the timing of the second peak was much more consistent, taking place shortly after impact.

The swing of one golfer was analysed further using high-speed images captured at 1000 frames per second with reflective tape used on the shaft. Six key points in the total grip force profile of the golfer were identified, each being a local maximum or minimum. These are indicated in Fig. 8, where the start of the downswing, impact and end of follow-through are also shown, and the golfer's position in the swing that corresponds to each of the six key points is displayed.

At Point 1, a local minimum, the golfer has brought the grip end of the club to nearly its full height in the backswing, just before the golfer starts the transition from backswing to the start of the downswing. The following peak, Point 2, occurs during the initial part of the downswing. Points 3 and 4 occur just on either side of wrist release during the downswing, and Point 5 is just after impact. Point 6 occurs during the follow-through after the right hand wrist cross-over, just as the left wrist begins to bend with radial deviation.

\subsection{Dynamic accuracy error analysis}

Previous analysis considered sensor performance and sources of error [17]. The most relevant source of error for this study is the dynamic accuracy of the sensors. The static error for the 9811 sensor was estimated at seven per cent while the dynamic accuracy error was much higher at around 60 per cent. This means that the height of short duration peaks in the 9811 data may be significantly diminished from the true height. For Flexiforce, the static error was slightly higher at 10 per cent but the dynamic accuracy was better with errors of approximately 20 per cent. This would still result in an underestimate of short duration peaks in the measurements, but to a lesser extent. Such error magnitudes may well be pessimistic, and the good comparison in Fig. 6 between 9811 and Flexiforce data provides some qualitative reassurance of the reliability of the data. It is also notable that the sample rate for the Flexiforce data was approximately four times higher than that with the 9811 sensor, and, for this reason, Flexiforce data might also give a better estimate of peak amplitudes.

\section{DISCUSSION}

Force data such as those provided here can be compared with the theories of professionals and coaches on how to grip the club. Several golf instruction books provide contradictory information on which hand should provide the firmer grip throughout the swing. Couples [19] and Nelson [20] have indicated that the left hand should grip firmer, while Faldo and Saunders [21] thought the right hand should have the tighter grip.

The total, left hand and right hand forces for each golfer in Figs 7(a) and (b) quantify the influence of each hand. For all 20 golfers that were tested, the left hand grip force exceeded the right hand force for the majority of the shot and, for most of the golfers, the left hand force was considerably larger than the right hand force around and during impact. It therefore appears that most golfers apply a larger grip force with their left hand throughout the shot.

As future work, the measurement systems described in this paper might be combined to contribute further to this debate. Resolving forces measured by the 9811 sensor on the grip in the direction of strike will reveal useful data on the forces accelerating the club, while simultaneous measurements on the hands using Flexiforce sensors would identify the regions of the hand responsible at any instant for the application of those forces to the club. 

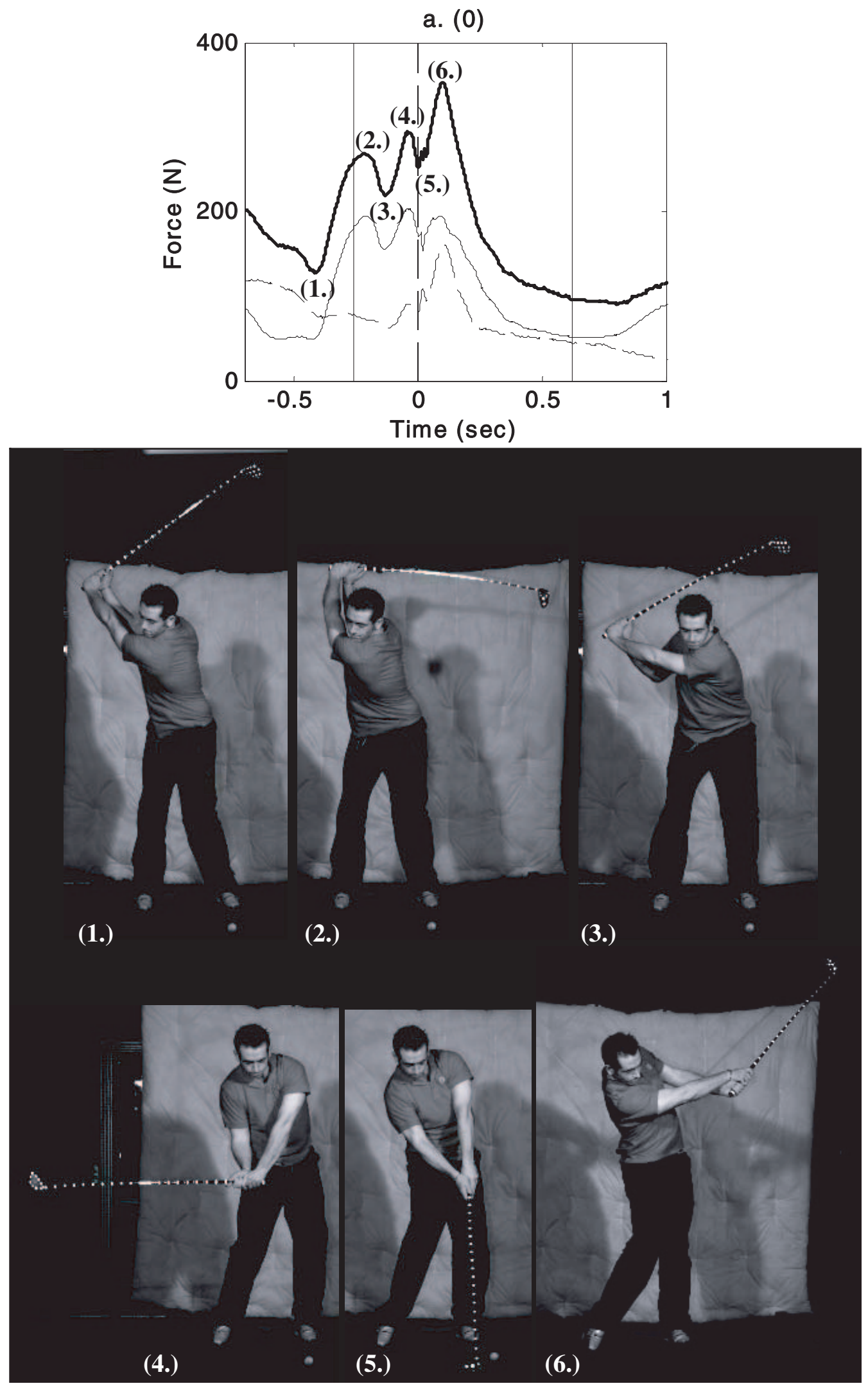

Fig. 8 Six key points on the mean total force curves for golfer a with corresponding still images from high-speed video footage; mean total (-), left hand (-), and right hand (---) forces 


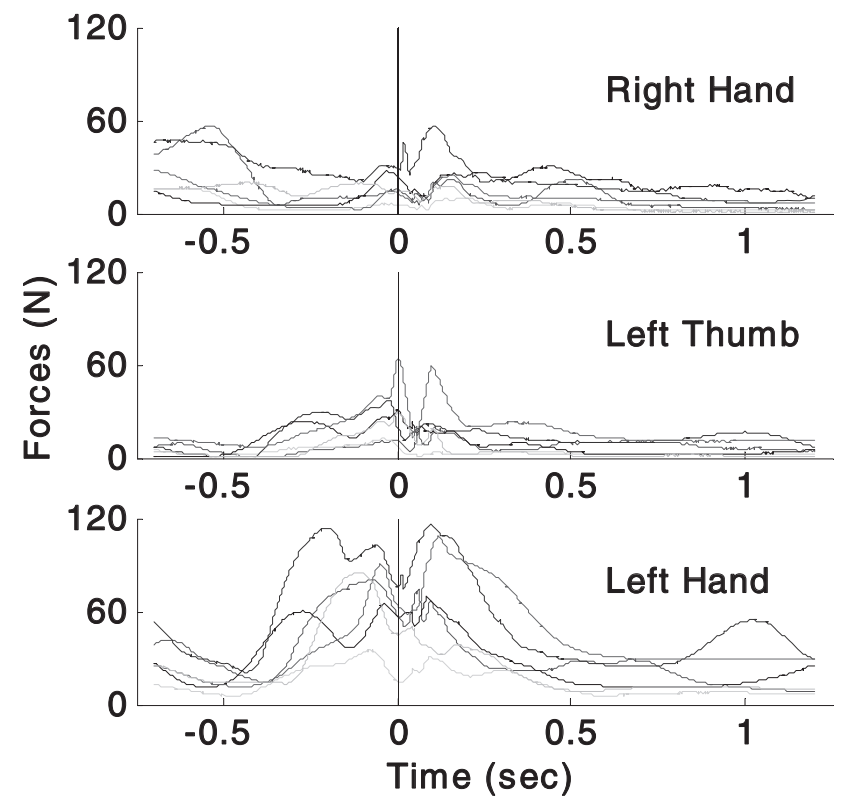

Fig. 9 Grip force from Flexiforce on gloves test in regions considered by Budney and Bellow, six category 1 golfers shown

A comparison can also be made between these tests and the data collected in previous golf grip force studies. As determined by Budney and Budney and Bellow [7, 8], relatively high loads were often recorded at the left thumb; however, these studies only provided data for a very limited number of golfers. It is important to note that in the current study there were only two force sensors on this digit compared to three on the other fingers, and the portion of the thumb in contact with the grip varied between players such that, for some golfers, the sensor may not have been able to collect the entire force applied by the thumb. In order to compare the forces produced in this study with those found by Budney and Bellow, the total forces in the three regions noted in the Budney and Bellow studies were calculated and plotted in Fig. 9 for six of the category 1 golfers that participated in this study. The right hand region was considered to be the palm sensors on the right hand, as well as the sensors under the proximal phalanges of the right index, middle, and ring fingers. The left thumb force was the summation of forces from the two thumb sensors, and the left hand region was considered to be all sensors under the middle, ring, and little finger of the left hand. The grip forces found in this study exhibited many of the same trends as the previous studies. The left thumb and hand forces were shown to have peak forces just before and after impact for most of the golfers, but, in this study, it did not appear that the right hand played such a large role prior to impact as seen in the Budney and Bellow studies. Differences in sensor area covered by the fingers will affect the magnitude of the forces measured, but comparable trends in the grip force for the golfers in both studies have been found.

\section{CONCLUSION}

Thin, flexible sensors were found to be suitable for this application of measuring time-resolved force during a golf shot. Grip force was measured on a golf club grip using a matrix sensor, and on gloves with single-cell sensors during a standard golf tee shot. The matrix sensors were very convenient for total force measurements, while individual sensors were preferred for examining force generated by localized regions of the hands. The existence of grip force 'signatures' was discovered, i.e. it was found that the forces produced by an individual golfer were very repeatable, but varied considerably between golfers. A cross-correlation was utilized to compare force traces between shots and between golfers to indicate similarities in gripping patterns. Trends did emerge, such as peaks in grip force just before and after impact, and an overall higher left hand force than right hand was measured for nearly all golfers tested. Comparisons conducted between data acquired with the two sensor types at several months apart emphasized both the validity of the data and the consistent nature of the signature. The distribution of force over smaller regions of the hands was also considered, with some comments made on trends that were found. Such detailed investigation of grip force can aid in future grip design, the creation of training aids, injury evaluation and prevention, and answer existing questions about how elite players actually grip the club.

\section{ACKNOWLEDGEMENT}

The authors would like to thank the golfers for their participation in this study.

\section{REFERENCES}

1 Hume, P. A., Keogh, J., and Reid, D. The role of biomechanics in maximising distance and accuracy of golf shots. Sports Medicine, 2005, 35(5), 429-449.

2 Hamill, J. and Knutzen, K. Biomechanical Basis of Human Movement, 2nd edn, 2003, p. 470, (Lippincott Williams \& Wilkins, Baltimore, MD).

3 Oatis, C.A. Kinesiology: The Mechanics \& Pathomechanics of Human Movement, 2004, p. 899, (Lippincott Williams \& Wilkins, Philadelphia, PA).

4 Hallbeck, M. S. and McMullin, D. L. Maximal power grasp and three-jaw chuck pinch force as a function of wrist position, age and glove type. Int. J. Industrial Ergonomics, 1993, 11, 195-206. 
5 Dempsey, P. G. and Ayoub, M. M. The influence of gender, grasp type, pinch width and wrist position on sustained pinch strength. Int. J. Industrial Ergonomics, 1996, 17, 259-273.

6 Mogk, J. and Keir, P. The effects of posture on forearm muscle loading during gripping. Ergonomics, 2003, 46(9), 956-975.

7 Budney, D. R. Measuring grip pressure during the golf swing. Research Quarterly, 1979, 50, 272-277.

8 Budney, D. R. and Bellow, D. G. Evaluation of golf club control by grip pressure measurement. In Science of Golf I, Proceedings of the World Scientific Congress of Golf, 1990.

9 Knudson, D. V. and White, S. C. Forces on the hand in the tennis forehand drive: application of force sensing resistors. Int. J. Sport Biomechanics, 1989, 5, 324-331.

10 Knudson, D. Forces on the Hand in the Tennis OneHanded Backhand. Int. J. Sport Biomechanics, 1991, 7, 282-292.

11 Stretch, R. A. A biomechanical analysis of the doublehanded grip forces. In Science and Racket Sports (T. Reilly, M. Hughes, and A. Lees, Eds), 1994, pp. 107-112.

12 Stretch, R. A., Buys, F. J., and Viljoen, G. The kinetics of the drive off the front foot in cricket batting: hand grip force. S.A. Journal for Research in Sport, 1995, 18(2), 83-93.

13 Stretch, R. A., et al. Kinematics and kinetics of the drive off the front foot in cricket batting. J. Sports Sciences, 1998, 16, 711-720.

14 Eggemen, G. W. and Noble, M. L. Design and testing of a baseball bat transducer. Experimental Techniques, 1985, 20-23, p. 20-23.

15 Nikonovas, A., et al. The application of force-sensing resistor sensors for measuring forces developed by the human hand. In Proc. IMechE, Part H: J. Engineering in Medicine, 2004, 218, 121-125.
16 Sprigings, E. J. and Neal, R. J. An insight into the importance of wrist torque in driving the golfball: a simulation study. J. Applied Biomechanics, 2000, 16, 356-366.

17 Komi, E. R., Roberts, J. R., and Rothberg, S. J. Evaluation of thin, flexible sensors for time-resolved grip force measurement. In Proc. IMechE, Part C: J. Mechanical Engineering Science, 2007, 221(12), 1687-1699.

18 Kong, Y.-K. and Lowe, B. D. Optimal cylindrical handle diameter for grip force tasks. Int. J. Industrial Ergonomics, 2005, 35, 495-507.

19 Couples, F. Total Shotmaking: The Golfers' Guide to Low Scoring, 1994, (HarperCollins Publishers, London).

20 Nelson, B. Byron Nelson's Winning Golf, 1947, (A. S. Barnes And Company, New York).

21 Faldo, N. and Saunders, V. Golf - The Winning Formula, 1989, (Lyons \& Burford, New York).

\section{APPENDIX}

\section{Notation}

$f_{m p} \quad$ force trace for subject $m$ and shot $p$

$\tilde{f}_{m p} \quad$ normalized force trace for subject $m$ and

shot $p$

$\tilde{F}_{m} \quad$ mean normalized force trace for subject $m$

$j, J$ data point and total number of data points, respectively

$P \quad$ total number of shots taken by a particular subject

$\hat{\gamma}_{n, m p} \quad$ peak cross correlation computed between the normalized mean force trace of subject $n$ and the normalized force trace for the $p$ th shot of subject $m$ 
